\title{
Metallic Nanoparticle: A Review
}

\author{
Harish Kumar K, Nagasamy Venkatesh*, Himangshu Bhowmik and Anuttam Kuila \\ Department of Pharmaceutics, A Constituent College of JSS University, India
}

Received: April 16, 2018; Published: April 27, 2018

*Corresponding author: Nagasamy Venkatesh, Department of Pharmaceutics, JSS College of Pharmacy, (A Constituent College of JSS University, Mysore), Ooty-643001, Tamil Nadu, India

\begin{abstract}
Metallic nano particle is nano sized metals with dimensions (length, width, thickness) within the size range of 1-100nm. In 1857, Faraday first investigated the existence of metallic nano particles in solution. In 1908, Mie gave a quantitative explanation of their colour. Today these nano materials can be prepared and modified with various chemical functional groups which allow them to bind with antibodies, ligands and drugs. Metallic nanoparticles give wide range of application in therapeutic area, biotechnology, vehicles for gene and drug delivery. This review summarizes the properties, advantages, disadvantages and characteristics of metal nanomaterials. This review also highlights on how metallic nanomaterials work as a catalyst and why is it necessary for stabilization. It provides the readers, detailed information on the synthesis by various methods, characterization, with particular focus on therapeutic application along with potential side effects and their future perspective. Recent headway had opened the way to site-specific targeting and drug delivery by these metallic nanoparticles.
\end{abstract}

Keywords: Metal Nanoparticle; Surface Atom and Quantum Dot; Catalyst; Gold; Platinum; Silver Nanoparticle

\section{Introduction}

From ancient time to the middle ages, the history of the nanoparticles has been summarized by Daniel and Astrum [1]. Because of their uniform size and sharp size distribution in nanometres; metallic nanoparticles have received much popularity. In the field of nanotechnology, metallic nanoparticles have shown number of properties and it has unlocked many new pathways in nanotechnology. Metallic nanoparticles have specialty with appropriate functional groups. It can be synthesized and modified that would allow them to bind with ligands, antibodies, drugs [2]. Metallic nanoparticle is nanosized metals with the size range of 10 $100 \mathrm{~nm}$. Metallic nanoparticles have unique characteristics such as surface Plasmon resonance and optical properties. Gold solution does have a golden yellow colour, for example, a solution of $20 \mathrm{~nm}$ gold nanospheres has red ruby colour where $200 \mathrm{~nm}$ nanospheres has bluish colour. The noble metals, especially silver and gold, have gained much attention to researchers in various branches of science and technology namely catalysis, photography, medical field as anticancer and anti-microbial agents. Faraday (1908) first recognized the existence of metallic nanoparticles in solution and Mie gave the quantitative explanation of their colour.

In medieval era, metallic nanoparticles were actually used to decorate cathedral windows. Due to unique properties of noble metal nanoparticles, it has made a special place in the field of nanotechnology. The most important feature of nanoparticles is their surface area to volume ratio, where it easily allows them to interact with other particles. In nanoparticles, high surface area to volume ratio makes diffusion faster and is feasible at lower temperatures. And this field has found more interesting, without disturbing and poisoning of healthy cells, we can directly treat affected cells and tissues. In fluorescence enhancement and surface enhanced Raman spectroscopy and in environment refractive index sensing nanoparticles have found additional application in the enhancement of field sensitive optical process. The optical properties of metal nanoparticles play a key role due to the localized surface Plasmon with resonance wavelength in the visible region. Silver and gold nanoparticles are effective in inhibiting growth of gram-positive and gram negative bacteria. For the production of nano devices, living organism has huge potential. However, it requires much more experimentation. There is a drawback such as involvement of toxic chemicals makes it difficult for synthesis of metallic nanoparticles.

So, there is an alternate way of synthesising metallic nanoparticles by using living organisms such as fungi, bacteria, plants. Several studies have shown that metallic nanoparticles characteristics like (size, stability, physical, chemical properties, morphology) are strongly influenced by the experimental conditions, adsorption process of stabilizing agent, the kinetics of interaction of metal ions with reducing agents. In various industrial 
applications, metallic nanoparticles have attracted, because of their different physical and chemical properties from bulk metals. Various properties like mechanical strengths, high surface area, low melting point, optical properties and magnetic properties. Catalysts which are used in metallic nanoparticles are selective and highly active, has long lifetime for many chemical reactions. It has experimented that a DVD disk with storage capacity of 10 tetra bytes, which are approximately 2000 movies of convectional size [3]. This is possible only due to the optical properties of gold nanorods embedded in the disk which are oriented randomly. To store data, Zijlstra and team used optical spectrum and different polarization directions.

The optical properties of gold, silver, lead, platinum nanoparticle arises from resonant oscillation of their free electrons in the presence of light, also known as Localized surface Plasmon resonance (LSPR). According to historical perspective, silver was considered as symbol of purity and it was valuable at that time as compare to gold. Since silver has many medicinal properties and it cures many diseases. It has anti-bacterial and antiseptic properties. In Ancient times, the noble metals were used to stained glass to produce the beautiful colors of drink cups such as Lycurgus cup [4] (Figure 1). In 1890, Robert Koch, the bacteriologist discovered that $\mathrm{K}[\mathrm{Au}(\mathrm{CN}) 2]$ potassium gold cyanide at low concentrations had anti-microbial activity against the Tubercle bacillus, from there onwards gold is introduced in modern medicine [5]. In 1727, John Herman Schulze first demonstrated that silver salts turned into black on exposure to light. In 1845, Michel Peyrone synthesized cisplatin (platinum containing anti-cancer drugs) [6]. In 1893, Alfred Werner elucidated the cisplatin structure whereas Roserberg studied the antitumor activity of cisplatin. In the 17th and 19th century, gold nanoparticles were used to treat fever and syphilis respectively [7].

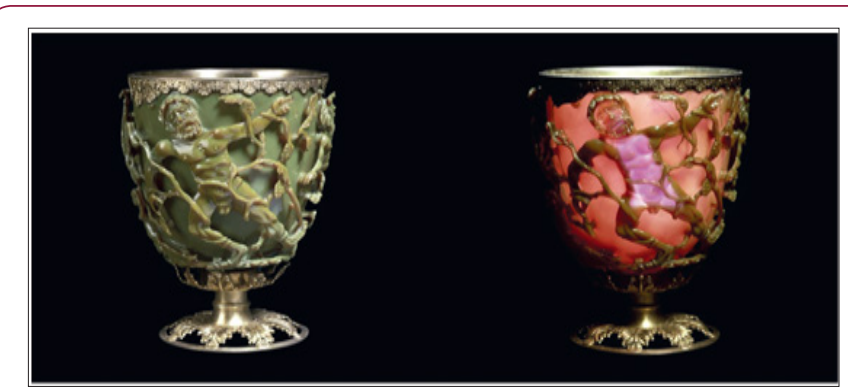

Figure 1: Photographs of the famous Lycurgus cup which displays a different colour depending on whether it is illuminated externally or internally. The British Museum, the Art of Glass, The Lycurgus Cup.

\section{Unique Properties of Metallic Nanoparticles}

\section{Surface Atom}

Percentage of surface atom can be explained from full-shell cluster structure that is arranged of atom which is corresponding to the magic number. Magic number is either protons or neutrons such that they are arranged into complete shells within the atomic nucleus. Magic numbers are developed from studies on rare gas clusters. Surface atom is reduced, if the clusters are bigger, because central atoms are surrounded by more atoms and those atoms which are in inner shell are covered by atoms in the upper shell. If clusters remain separated from other clusters it is called as Dispersed clusters and if these clusters joint together it is called as aggregated clusters. If atoms is in equal amount both in two clusters (dispersed \& aggregated), the dispersed clusters have higher surface area than the other. The rates of chemical reaction are affected by surface area of reactant. So, the reactant composing of dispersed clusters shall have higher reactivity than the aggregated clusters.

\section{Quantum Dot}

These are very tiny particles which are in nanometre range. They are made of thousands of atoms. These semiconductor materials can be made from silicon or Germanium. So, quantum dot can be explained through Band theory in semiconductor. Band gap refers to the energy difference between the valence band (top) and conduction band (bottom). Metals that are categorized as semiconductor electronically have partially filled band separated from the empty conduction band by a band gap. When we are dealing with interacting molecular orbital, the two that interact are generally HOMO and LUMO. HOMO (The Highest energy occupied molecular orbital) of one molecule. LUMO (The lowest energy unoccupied molecular orbital) of the other molecule. HOMO \& LUMO are the pair that lies close in energy of any pair of orbital's in the two molecules, which allow them to interact strongly. These orbitals are also called as Frontier orbital, because they lie at the outermost boundaries of electrons of the molecules. Therefore, quantum mechanics are applicable in describing the energy of the metal nanoparticles.

\section{Advantages of Metallic Nanoparticle}
a. Enhance Rayleigh scattering
b. Surface enhanced Raman scattering
c. Strong plasma absorption
d. Biological system imaging
e. Determine chemical information on metallic nanoscale substrate [8].

\section{Disadvantages of Metallic Nanoparticles}

a. Particles instability: Nanomaterials can undergo transformation, as they are thermodynamically unstable and lie in the region of high energy local minima. This leads to deterioration of quality, poor corrosion resistance, and main concerned is retaining the structure becomes difficult.

b. Impurity: While synthesising nanoparticles, nitrides, oxides, formation can aggravated from the impure environment. As nanoparticles are highly reactive, there can also be high chances of impurity as well. In solution form, nanoparticles should be synthesized in form of encapsulation. So, it becomes a challenge to overcome impurity in nanoparticles.

c. Biologically harmful: nanomaterials has been reported toxic, carcinogenic and cause irritation as they become transparent to the cell dermis. 
d. Explosion: exothermic combustion can lead to explosion, as fine metal particles act as strong explosives.

e. Difficulty in synthesis: while synthesizing nanoparticles, it should be encapsulated, because it is extremely challenging to retain the nanoparticles size in solution form [9].

\section{Characteristics of Metallic Nanoparticles}

a. Large surface energies

b. As compared to bulk they have large surface area to volume ratio
c. Quantum confinement
d. Plasmon excitation
e. Increased number of kinks [10].

\section{Points Need to Be Considered While Preparing Metallic} Nanoparticles

Ideally, metallic nanoparticle should be prepared by a suitable method which is
a. Easily reproducible
b. Easily available, economical
c. Use minimum number of reagents
d. May control the particle shape
e. Use a reaction temperature close to room temperature
f. Minimizing the quantities of generated by-products and waste.

\section{Metal Nanoparticles as Catalyst}

It is already known that metallic nanoparticle function as a catalyst for various kinds of chemical reactions. On its surface metals catalytic sites are located. So, by this we can mean that metallic nanoparticles of $1-10 \mathrm{~nm}$ size can work as effective catalysts. With respect to all atoms in a particle the ratio of surface atoms increases with decreasing particle size. Metallic nanoparticles should be stabilized under the catalytic condition, when it is used as catalysts. Otherwise what will happen is, it will easily coagulate in solution and leads to form aggregates, which are less effective as catalysts. Advantages of using metal nanoparticles as catalysts are the following:

a. The temperature applied to the catalyst where the metallic nanoparticles dispersed in solution is below the boiling point of the solvent.

b. Metallic nanoparticles dispersed in solutions can be used as photo catalysts as they are transparent to light.

c. By the preparation, metallic nanoparticles size and shape can be easily controlled.

d. Metallic nanoparticles immobilized on solid supports acts as catalysts even for the reactions in a gaseous phase. e. Bimetallic and trimetallic nanoparticles can be prepared by modifying the structures and compositions.

\section{Stabilization of Metallic Nanoparticles}

With large surface energy nanoparticles coalesce to each other to give thermodynamically favoured bulk particle. Coagulation will occur between two metallic nanoparticles in the absence of repulsive forces. So, it is essential to stabilize the metallic nanoparticles for spatial confinement of the particles in nano range. So, this stabilization can be achieved either by steric exclusion or electrostatic stabilization by using a capping agent such as surfactant, polymer, solid support or ligand with suitable functional groups.

\section{Electrostatic stabilization}

According to DLVO theory, the total interaction between two particles, which are stabilized electrostatic, is the combination of Vander Waals attraction and electrostatic repulsion (Figure 2a).

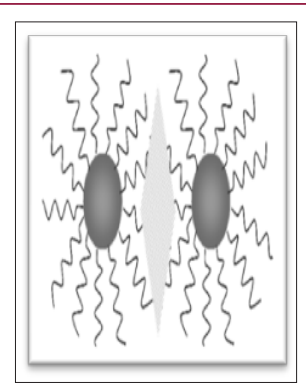

Figure 2: (a) Electrostatic stabilization of nanostructured metal colloids, (b) Steric stabilization of nanostructured metal colloids.

\section{$\mathrm{F}=\mathrm{Fa}+\mathrm{Fr}$}

There are some assumptions in the DLVO theory- uniform surface charge density, no change of concentration profiles of both counter ions and surface charge determining ions, i.e., the electric potential remains unchanged, infinite flat solid surface. However, in spite of the assumptions, the DLVO theory works very well in explaining the interaction between two approaching particles, which are electrically charged, and thus is widely accepted in the research community of colloidal science.

Some limitations of electrostatic stabilization are

a. It is a kinetic stabilization method

b. Only applicable to dilute systems

c. Not applicable to electrolyte sensitive systems

d. Difficult to apply to multiple phase systems, since in a given condition, different solids develop different surface charge and electric potential.

\section{Steric stabilization}

Also called as polymeric stabilization. This method is widely used in stabilization of colloidal dispersions. However, it has several advantages over electrostatic stabilization[Fig.2b] 
i) It is not electrolyte sensitive

ii) It is suitable to multiple phase system

iii) It is a thermodynamic method, so that the particles are always re-dispersible.

By binding of polymer with long alkyl chains to the particle surface, steric stabilization is achieved [11].

\section{Synthesis of Metallic Nanoparticles}

There is two methods for the synthesis of metallic nanoparticles- top-down and bottom-up. Bottom up approach [11]. These approaches include the attenuation of materials components with further self-assembly process which leads to the formation of nanostructures. During self-assembly the physical forces operating at nanoscale are used to combine units into large stable structures. Typical examples include Quantum dot and formation of nanoparticles from colloidal dispersion. Top down approach- These approaches include macroscopic structures which can be externally controlled in the processing of nanostructures. Typical examples are ball milling, application of severe plastic deformation. Top down method v/s bottom up methods- Top down method starts with a pattern generated on a large scale, then reduced to nanoscale, quick to manufacture, slow and not suitable for large scale production. Bottom-up approach- begins with atoms or molecules and build up to nanostructures, fabrication is much less expensive. Attrition/ milling is top-down type of method and bottom-up method is production of colloidal dispersion.

\section{Chemical Reduction Method}

\section{Gold Nanoparticle}

For centuries, gold nanoparticles have been intensively studied. When chitosan capped gold nanoparticles coupled with ampicillin, two-fold enhancement of antibacterial activity was observed [12] Chemical reduction is the most common and widely used method for the preparation of gold nanoparticles. This method includes, in the presence of reducing agent, gold salt is reduced [13]. In 1857,
Michael Faraday first studied gold colloids synthesis in solution phase, where in an aqueous medium gold chloride reduced with phosphorus [14]. One article has been reported in 1951 which discovered citrate reduction method [15]. Synthesis of AuNPs was based on a single phase reduction of gold tetrachloroauric acid by sodium citrate in an aqueous medium and produced particles about 20nm [16]. The major contribution for AuNPs synthesis was published in 1994 and now it popularly known as BurstSchifrin method [17]. This process use two phases that exploits thiol ligands that strongly bind to gold due to the soft character of S and Au. Firstly, with the help of a phase transfer agent such as tetraoctylammonium bromide gold salt is transferred into an organic solvent, then organic thiol is added. At last, strong reducing agent such as sodium borohydride (in excess) is added which gives thiolate protected AuNPs.

The major benefit of synthesizing this process are ease of preparation, size controlled, thermally stable nanoparticles, reduced dispersity [18]. Natan investigated gold nanoparticles seeded growth by using modification on the Frens synthesis [19]. Bastus have synthesized monodispersed citrate stabilized particles through kinetically controlled seed growth [20]. Narrow size distribution was prepared with a uniform quasi spherical shape (up to $200 \mathrm{~nm}$ ) which is kinetically controlled seeded growth strategy via HAuCl4 reduction by sodium citrate. During homogeneous growth, the inhibition of any secondary nucleation was controlled by adjusting temperature, $\mathrm{pH}$, and seed particle concentration. Frens method improved results in different aspects-

i. It allows better control of size and size distribution of gold nanoparticles

ii. It produces particles of higher monodispersity

iii. It leads to higher concentration.

With a wide variety of molecules, this method can be further functionalized. Hence, this method seems to be promising in the biomedicine, electronics and photonics fields (Figure 3).

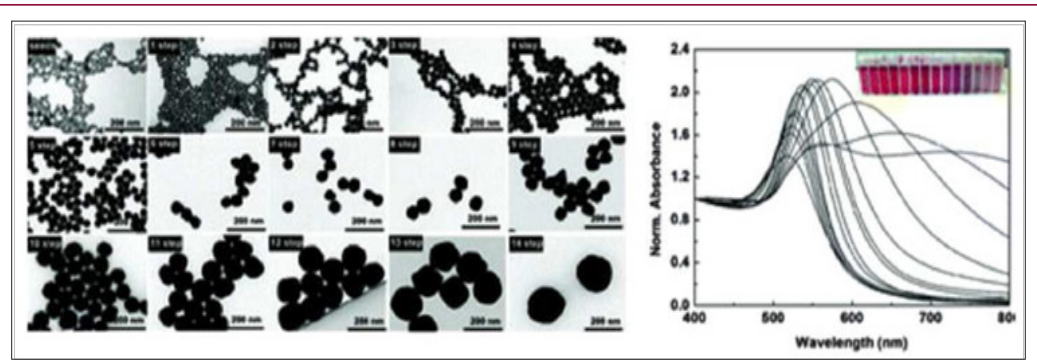

Figure 3: Monodispersed citrate-stabilized gold nanoparticles [Kinetically controlled seeded growth synthesis of citratestabilized gold nanoparticles of up to $200 \mathrm{~nm}$ : size focusing versus Ostwald ripening].

\section{Platinum Nanoparticles}

In the synthesis of platinum nanoparticles, the platinum metal precursor either in an ionic or a molecular state is taken. By the reducing agents, chemical changes are made to convert the precursor to platinum metal atoms. These metal atoms then combine into stabilizer or supported materials to form nanoparticles. For example, in chemical reduction $\mathrm{H} 2 \mathrm{PtCl} 6$ is reduced by $\mathrm{NaBH} 4$ or $\mathrm{Zn}$ to give rise to platinum nanoparticles. $\mathrm{H} 2 \mathrm{PtCl} 6+\mathrm{NaBH} 4=$ $\mathrm{Pt}+$ other reaction product $\mathrm{H} 2 \mathrm{PtCl} 6$ is the common precursor used in synthesis of platinum nanoparticles. $\mathrm{H} 2 \mathrm{PtCl} 6$ is usually dissolved in organic liquid phase or aqueous phase. By introducing decomposition, displacement, reducing agent, electrochemical 
reactions, the dissolved metal precursor can be converted into the solid metal. Radiolytic, sonochemical or electrochemical method by this three method, chemical step can be activated by physical mixing. Two different reactivites are generally used in case of mixed metal nanoparticles e.g., $\mathrm{RuCl} 3$ and $\mathrm{H} 2 \mathrm{PtCl} 6$ \{Na6Pt (So3)4, Na6Ru (So3)4 $\{$ PtCl2 and $\mathrm{RuCl} 3$ ) $\}$ various complex mixed precursors have been also used.

\section{Silver Nanoparticles}

AgNps are one of the most attractive inorganic materials because of its environment free nature [21]. Moreover, it has several applications in various field like in photography, diagnostics [22], catalysis [23], biosensor [24], antimicrobial [25,26].

\section{a. Reduction by citrate anion}

From the pioneering studies; it is now well known that citrate acted in both ways. First is to stabilize the nanoparticles and to reduce the metal cation. To determine the particles growth this reactant played a major role. Citrate controls the size and shape of AgNps. This function was investigated by Pillai and kamat. At different citrate concentration, by using the boiling method, AgNps with Plasmon maximum absorbance at $420 \mathrm{~nm}$ were produced. By increasing the concentration 1 to 5 times of sodium citrate to silver cation. i.e., [citrate] $/[\mathrm{Ag}+]$, the elapsed time for AgNps formation was 40 to $20 \mathrm{~min}$ reduced respectively, which indicates that a fraction of the $\mathrm{Ag}+$ was not reduced under equimolar conditions.

\section{b. Reduction by Gallic acid}

At room temperature, reduction of Ag+ in water can be achieved by using Gallic acid (GA) whose oxidation potential is $0.5 \mathrm{~V}$. In benzoic acid structure the hydroxyl group at determined positions plays an important role in the synthesis of metal nanoparticles. When hydroxyl groups are located at Meta position, nanoparticles synthesis was not successful but it was achieved when hydroxyl groups are present at ortho and para positions. Here carboxylic group act as stabilizer and hydroxyl as the reactive part. To obtain silver colloids, $\mathrm{NaOH}$ addition is important. Then, the silver species reacting could be Ag2O that has been reported as a good AgNps precursor by thermal decomposition.

\section{Physical Method}

To form AuNPs, photochemical reduction of gold salts has been used [27]. This formulation uses continuous wave UV irradiation (250-400nm), PVP as the capping agent, ethylene glycol as the reducing agent. Glycol concentration and viscosity of the solvent mixture are the two factors where AuNPs formation is dependent upon. Process was further improved by the addition of $\mathrm{Ag}+$ to the solution, leading to an increase in the production of Au nanoparticles [28]. To synthesize platinum nanoparticles, irradiation and laser ablation techniques have also been used. In one method irradiation was combined with ultrasonication. So, in this process, $\mathrm{H} 2 \mathrm{PtCl} 6$ $6 \mathrm{H} 20$ was added to a solution of $10 \mathrm{~mm}$ polypyrole and SDS. Particle size is controlled by varying the length and time of ultra sonication and irradiation [29].

\section{Biological Method}

Plant mediated synthesis has gained more popularity due to eco-friendly. Zingiber Officinale extract acts as a reducing agents, as well as stabilizer with particles ranging from $5-15 \mathrm{~nm}$ in diameter. To synthesize metallic nanoparticles, several bacteria and fungus like prokaryotic bacteria and eukaryotic fungus. Plant extract may have been employed for the reduction of aqueous metal ions. Biological methods may have wide distribution in particle size but have a slow reaction rate. At room temperature, the extract is mixed with a metal salt solution, within minutes the reaction is complete. By this method, gold, silver nanoparticles have been synthesised. The rate of nanoparticles production, their quantity can be affected by the plant extract concentration, its nature metal salt concentration, temperature, the pH. By using a leaf extract of Polyalthia longifolia, silver nanoparticles were synthesised, which was reported by Prasad and Elumalai (2011). For preparing metallic nanoparticle by the use of plant extract is environmental friendly, economical. It brings controlled size and morphology of nanoparticles (Figure 4).

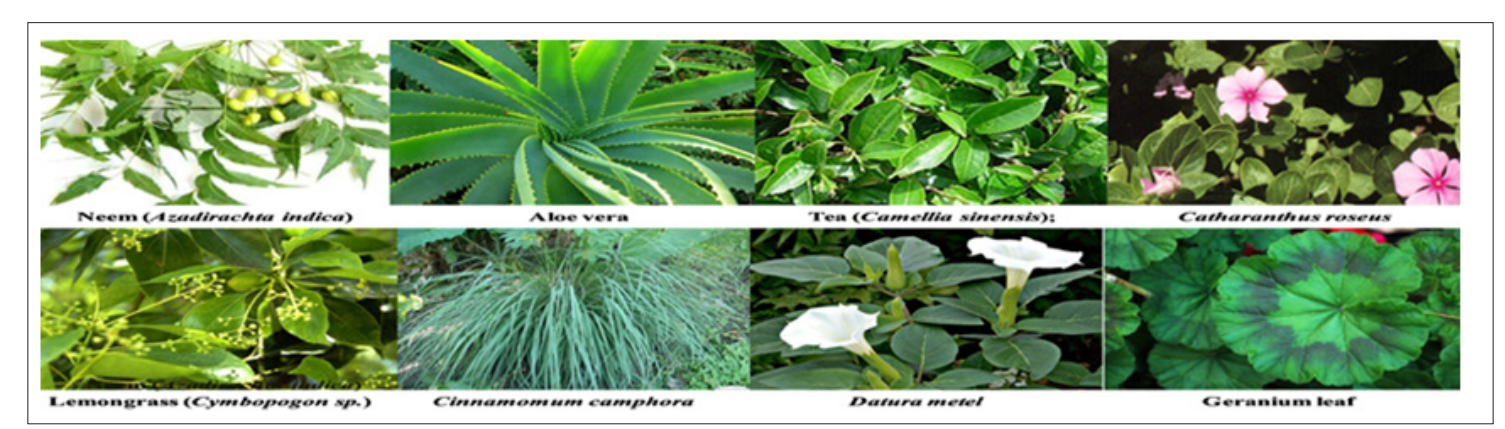

Figure 4: Various types of plants used for the synthesis of metal nanoparticles.

\section{Characterization of Metallic Nanoparticle}

a) Absorbance Spectroscopy: Spectroscopy is useful to characterize metal nanoparticles, because they possess bright colour which is visible by naked eye. By this technique, qualitative information about the nanoparticle can be obtained.
By applying Beer's law absorbance is measured. Depending on path length (l), nanoparticle conc. (c), extinction coefficient (A) can be obtained.

b) Infrared Spectroscopy: This method can provide information on organic layers surrounding metallic 
nanoparticles. It also gives valuable information to understand the surface structure of the metal nanoparticles.

c) TEM: (Transmission electron microscope) is also widely used to characterize nanomaterials to gain information about particle size, shape, crystallinity and interparticle interaction. TEM is a high spatial resolution structural and chemical characterization tool. It has the capability to directly image atoms in crystalline specimens at resolutions close to $0.1 \mathrm{~nm}$, smaller than interatomic distance. An electron beam can also be focused to a diameter smaller than $\sim 0.3 \mathrm{~nm}$, allowing quantitative chemical analysis from a single nanocrystal.

d) SEM: (Scanning Electron Microscopy) It is a powerful technique for imaging any material surface with a resolution down to about $1 \mathrm{~nm}$. The interaction of an incident electron beam with the specimen produces secondary electrons, with energies smaller than 50ev. SEM can give information about the purity of nanoparticle sample.

e) AFM: It is a better choice for nonconductive nanomaterials. Typically, it has vertical resolution of less than $0.1 \mathrm{~nm}$ and lateral resolution of around $1 \mathrm{~nm}$.It gives detailed information on the atomic scale, which is important for understanding electronic structure and chemical bonding of atoms and molecules.

f) XRD: It is useful and widely used technique for determining the crystal structures of crystalline materials. Diffraction line widths are closely related to the size and their distribution, strain in nanocrystal. The line width is broadened, as the size of the nanocrystal decreases, due to loss of long range order relative to the bulk. XRD line can be used to determine the particle size by Debye-Scherrer method.

$\mathrm{D}=0.9 \lambda / \mathrm{bcos} \theta$ Where,

$\mathrm{D}=$ nanocrystal diameter

\author{
$\lambda=$ light wavelength \\ $\mathrm{b}=$ full width half at max. Of the peak (radians) \\ $\theta=$ Bragg angle
}

g) FTIR: It is widely adopted techniques compared to IR spectroscopy. Functional groups attached to the metallic nanoparticle surface show different FTIR pattern than those of free groups.

h) EXAFS: (Extended X-ray Absorption Fine Structure) this is one of the most reliable and powerful characterization technique to evaluate the structure of metallic nanoparticles; especially it is useful to determine bimetallic nanoparticles. To gain appropriate information about the structure, the sample of metallic nanoparticles should be homogeneous. This method provides the no. Of atoms surrounded the x-ray absorbing atom and their interatomic distances involved in the shells.

i) XPS: (X-ray Photoelectron Spectroscopy) it is used to provide information on metal state. Suppose the oxidation state of metal on the surface. It is often oxidized by air. So, by using this method, 0 -valency of surface metal must be confirmed.

\section{General Application of Metallic Nanoparticles \\ Optical Function}

Imaging sensor, display, solar cell, Photo catalysis, biomedicine, optical detector, laser-this are the applications based on the optical properties of metal nanoparticles. It is mainly dependent on some factors such as shape, size, surface area, doping, and interaction with the surrounding. The optical properties of CdSe semiconductor nanoparticles can change with size. For different samples of gold nanospheres, the optical properties changes with enlargement of metallic nanoparticles. Surface absorption Plasmon Au \& Ag can change into various colors by changing the particle size, form and shape of the particle and condensation rate (Figure 5).

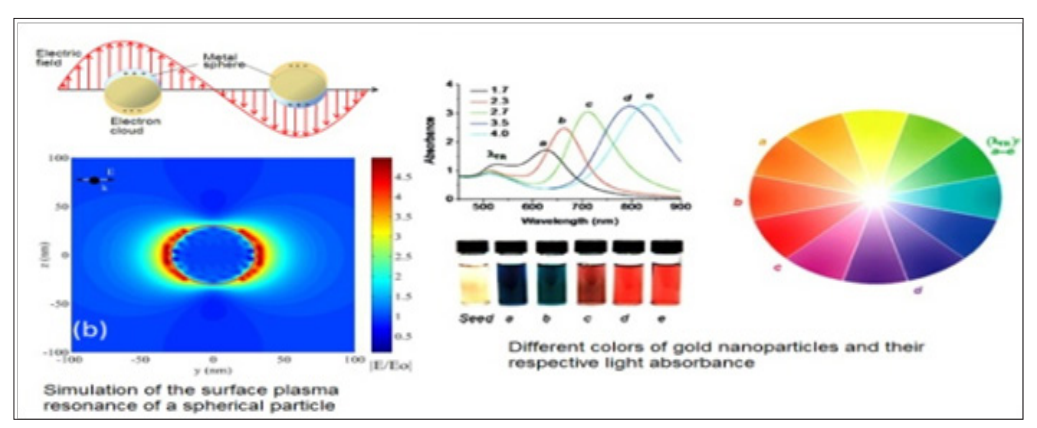

Figure 5: The optical properties of the nanoparticles changes, when anisotropy is added to the nanoparticle, changes such as nanorods growth.

\section{Thermal Function}

When nanoparticle diameter is less than $10 \mathrm{~nm}$, the melting point is also lower than a bulk metal. With low boiling point, electronic wiring can be made with nanoparticles.

\section{Electrical Function}

Can be used to make high temperature superconductivity material. In conductance one step can be shown, at constant applied voltage, the mechanical thinning of a nanowire and electric current measurement. So the main point here to be noted is that, the number of electron wave modes supporting to the electrical conductivity is becoming smaller with decreasing diameter of the wire. Only one electron wave mode is observed in electrically conducting carbon nanotubes which transport the electrical current. Electrically conducting carbon nanotubes touch the mercury surface at different times, as their length and orientation are different and this leads to transport of electrical current. This 
gives two type of information i) different nanotubes resistance ii) the effect of carbon nanotubes length on the resistance.

\section{Mechanical Function}

Polymers filled with nanotubes leads to improvement in their mechanical properties. And this progress is purely dependent on the filler type and the way with which the filing is conducted. The larger the particle size of the filler, poorer is the properties obtained. Polymer matrix and defoliated phyllosilicates consisting components provide excellent mechanical properties. Mechanical property of metallic nanoparticles can be improved by mixing the nanoparticles with metals or ceramics.

\section{Magnetic Function}

At the nanosized level, pt and gold nanoparticles exhibit magnetic property but as bulk they are non-magnetic. By capping, the nanoparticle surface and bulk atoms can be improvised by interaction with other chemical species. So, by capping with appropriate molecules; this gives the chances to modify the physical property of nanoparticles.

\section{Catalysis}

Catalysts based on metallic nanoparticles are-selective, highly active, exhibit long lifetime for several kinds of reactions. So, there are two types of catalyst- Heterogeneous catalysts- which are immobilized on inorganic support. Applications- Oxidation reactions, synthesis of $\mathrm{H} 2 \mathrm{O} 2$, water gas shift, hydrogenation. Homogenous catalysts- metallic nanoparticles surrounded with stabilizers. Applications- Nitrile hydrogenation, olefin hydrogenation.

\section{Used as Fuel Cell Catalysts}

Fuel cell is a device that directly converts chemical potential energy into electric energy. A PEM (Proton Exchange Membrane) cell uses hydrogen gas (H2) \& oxygen gas (02) as fuel. The products of fuel cell are water, electricity, heat.

\section{Used in Materials Science}

Nickel nanoparticles are used as electrical conductive pastes, battery materials etc.

\section{Used in Medical Treatment}

Healthy cell can be distinguished from cancer cell by the presence of Antibodies joined to the Au nanoparticle.

\section{Used in Paints}

Nano titanium dioxide is used in paint to exploit two outstanding properties- photo catalytic activity, UV protection. Addition of nanosilicon dioxide to paints can improve the macro and micro hardness, abrasion, scratch resistant.

\section{Elimination of Pollutants}

As metallic nanoparticles is highly active in terms of physical, chemical and mechanical properties. They can be used as catalysis to prevent environment pollution arising from coal and burning gasoline. As they react with toxic gases such as carbon monoxide and nitrogen oxide.

\section{Used as Sun Screen Lotion}

Nanomaterials are very useful as sunscreen lotions by blocking UV radiation effectively for a prolonged period of time. As prolonged UV exposure causes skin burns. By applying sun-screen lotions containing nano-Tio2 it gives sun protection factor (SPF).

\section{Therapeutic Applications of Metallic Nanoparticles As anti-Infective Agents}

The anti-viral properties of AgNps are more effective then chemically synthesized silver nanoparticles [30]. In one study, metallic nanoparticles have been described as a HIV preventative therapeutic [31]. In a couple of studies, it has been shown that as virucidal agent silver acts directly on the virus by binding to the glycoprotein gp120 [32]. This binding in turn prevents the CD4 dependent virion binding which effectively decreases HIV1's infectivity [33] and it has also been reported that metallic nanoparticles has been effective antiviral agents against herpes simplex virus [34], influenza [35], respiratory syncytial viruses [36].

\section{As anti-Angiogenic}

It is well known that angiogenesis is the development of new blood vessels and occurs during normal development and in some disease states. It plays a main role in number of diseases such as cancer, rheumatoid arthritis. In normal conditions, angiogenesis is tightly regulated between various pro-angiogenic growth factors (VEGF, PDGF, and TGF-B) and anti-angiogenic factors (platelet factor 4, TSP-1). Under diseased conditions, angiogenic is turned on. Some reviews have reported that these agents have serious toxicities such as fatal haemorrhage, thrombosis, and hypertension. It may be overcome if these nanoparticles alone can be efficacious as an anti-angiogenic agent.

\section{In Tumour Therapy}

It has been studied that naked gold nanoparticles inhibited the activity of heparin-binding proteins such as VEGF165 and bFGF in vitro and VEGF induced angiogenesis in vivo [37]. Further work in this area has been reported that onto the surface of AuNPs heparin binding proteins are absorbed [38] and were subsequently denatured[39].The researchers also showed that surface size plays a main role in the therapeutic effect of AuNPs. Mukherjee and colleagues also experimented the effect of gold nanoparticles on VEGF mediated angiogenesis using a mouse ear model injected with an adrenoviral vector of VEGF (Ad-VEGF- mimics the resulting angiogenic response found in tumours)[40]. A week later, the AdVEGF administration, mice treated with AuNPs developed lesser edema than the same treated mice. Eom and Colleagues revealed the anti-tumour effects of 50nm AgNps In vitro and In vivo.

\section{In Multiple Myeloma}

Researchers (Washington university school of Medicine in St.Louis, journal Molecular cancer Therapeutics) have designed a nanoparticle based therapy that is effective in treating mice with multiple myeloma. Multiple myeloma is a cancer that affects plasma cells. Mukherjee and group demonstrated that a gold nanoparticle 
inhibits the VEGF and bFGF dependent proliferation of multiple myeloma cells.

\section{In Leukaemia}

B-chronic Lymphocytic Leukaemia (CLL) is an incurable disease predominantly characterized by apoptosis resistance, by co-culture with an anti-VEGF antibody, found induction of more apoptosis in CCL B cells. In CLL therapy, gold nanoparticles were used to increase the efficacy of these agents. Gold nanoparticles were chosen based on their biocompatibility, very high surface area, surface functionalization and ease of characterization. To the gold nanoparticles, VEGF antibodies were attached and determined their ability to kill CLL B cells.

\section{In Rheumatoid Arthritis}

Scientists from the University of Wollongong (Australia) have built a new class of anti-arthritic drug which could be used by gold nanoparticles and it has fewer side effects. Rheumatoid arthritis is an autoimmune disease that occurs when the immune system not function properly and attacks a patient's joints. New research has shown that gold particles can invade macrophages, and stop them from producing inflammation without killing them. Journal of inorganic biochemistry it has been published that by reducing the size of gold into smaller nanoparticles $(50 \mathrm{~nm})$ was able to cause more gold to immune cells with lesser toxicity.

\section{In Photo Thermal Therapy}

Gold nanoparticles absorb light strongly as they convert photon energy into heat quickly and efficiently. Photo-thermal therapy (PTT) is an invasive therapy in which photon energy is converted into heat to kill cancer.

\section{In Radiotherapy}

Tumours loaded with gold, this absorbs more X-rays as gold is an excellent absorber of X-rays. Thus deposition of more beam energy and results in a local dose which increase specifically to tumour cells. Gold nanoparticles have been more useful to treat cancer.

\section{Potential Side Effects of Metal Nanoparticles}

Argyria is one of the reported side effects in patients which results from prolonged contact with or ingestion of silver salts. Argyria is characterized by gray, black staining of the skin and mucous membrane produced by silver deposition. Silver may be deposited in the skin either from silver salts containing medications or from industrial exposure. One report was suggested where a patient ingested colloidal silver 3 times a year over a 2year period resulted in diabetics, hypertension, hyperlipidaemia [41]. Mouse brains exposed to nanosilver reported apoptosis and gene modulation [42]. Due to inhalation, workers also had tarnished corneas and conjunctiva [43]. Without any reported side effects, gold colloid has been used for centuries in therapeutic field. It has also been reported that gold nanoparticle causes thrombosis, immunogenic reactions, and haemolysis [44]. Enzyme present in saliva can transform gold (0) to gold (I), which is subsequently engulfed by immune cells.
Some side effects related to this are erythema nodosum, allergic reactions, macular and papular rash. While injected with gold complexes leads to very low incidence of nephrotoxicity with minor proteinuria. Due to teratogenicity of gold complexes it is not recommended for pregnant women because it can leads to haematological disorder. In healthy human body, gold is present in the range of $0-0.001 \mathrm{ppm}$ [45]. It is found in small amounts in skin $(0.03 \mathrm{ug} / \mathrm{g})$, hair $(0.3 \mathrm{ug} / \mathrm{g})$ and nails $(0.17 \mathrm{ug} / \mathrm{g})$ [46-49]. It has been reported that $\mathrm{Au}, \mathrm{Ag}$ and $\mathrm{Pt}$ nanoparticles toxicity over 72 hour period using Zebrafish model. It causes delayed hatching, crippled backbone, cardiac disorders, and platinum accumulation in brain when they found polyvinyl alcohol capped at particles (3$10 \mathrm{~nm}$ ).It has been reported that the neurotoxicity of $\mathrm{Cu}$ and $\mathrm{Mn}$ nanoparticles in PC-12 cell lines.

\section{Metallic Nanoparticle as Drug Delivery}

Most of the chemotherapeutics agents distribute to the whole body results in toxicity and it gives poor compliance by patients, so as targeted delivery of therapeutic agents to tumour cells is a challenge. By active and passive targeting, imaging of tumour cells is done by metallic nanoparticles. Both at surface and inside cells, metallic nanoparticles can interact with bio molecules because of their small size which gives better targeting for therapeutics. Between 10-100nm [50] of different shapes, sizes of gold, nickel, silver, iron [51] metallic nanoparticles have been checked out as diagnostics and drug delivery systems. Gold nanoparticles utility in cancer cells and in xenograft tumour mouse models was experimented and reported the use of non-toxic PEG gold nanoparticles for tumour targeting (in vivo) which were biocompatible and were characterized by SERS (surface enhanced Raman scattering) [52]. But the use of metallic nanoparticles for drug delivery is a concern because after drug administration, some fraction of metallic particles can be retained in the body even though it is inert and biocompatible.

These metallic nanoparticles can be easily conjugate with various agents such as peptides, antibodies and DNA/RNA to specifically target different cells [53], with polymers (polyethylene glycol) which are biocompatible to prolong their circulation in vivo for drug and gene delivery applications [54-55]. They can also transform light into heat, thus enabling thermal ablation of targeted cancer cells [56,57]. For the delivery of anticancer drugs such as Paclitaxel [58] or cisplatin, oxiplatin (platinum based drugs); $\mathrm{Au}$ nanoparticles have been used as vehicles. This has investigated $2 \mathrm{~nm}$ $\mathrm{Au}$ nanoparticles covalently bind with the chemotherapeutic drug paclitaxel. Gold-gold sulphide nanoshells have been produced as a photo thermal modulated drug delivery system. These nanoshells covered by a hydro gel matrix which are thermo sensitive. These nanoshells were basically designed to strongly absorb NIR light and to release multiple bursts of any soluble material held within the hydro gel matrix in response to repeated NIR irradiation. By using, 50nm hollow Au-nanocubes with eight lopped off porous corners which are covered by a thermo sensitive polymer containing preloaded effectors that can be released in a controllable manner using an NIR laser. This work was studied by Yavuz and team. 
Protein modified 10, 20, and 40nm Au nanoparticles and 20, 50, and $100 \mathrm{~nm}$ Ag nanoparticles which are obtained from fetal bovine serum have potential effects on radiation induced killing of glioma cells. This work was studied by Xu and co-workers.

\section{Conclusion}

Metallic nanoparticles in this 21st century are highly demanded because it can be synthesised through various routes. Metallic nanoparticles synthesis is important because of their ideal electrical, optical, magnetic and chemical properties. And it has been proved that by Radiolytic and photolytic methods it offers several advantages, as it is easy to synthesize nanoparticles of narrow size distribution. Moreover, ultrafast lasers and electron pulse radiolysis can be employed in combination for nanoparticles preparation. The novelty of this technique is, along with the formation of metal nanoparticles, growth kinetics can also be studied. Recently, seed method was used to synthesize bigger size uniform particles. $\mathrm{Cd}$ and Ag metal nanoparticles were prepared in viscous medium and in aqueous solutions. To stabilize metal nanoparticles viscous medium was used.

Uniform particle size can be prepared by bombarding the particles by laser irradiation in ethylene glycol and glycerol media. Metallic nanoparticles have wide range of applications such as imaging agents, delivery vectors, synthetic inhibitors and sensors. In nanobiotechnology, one of the major challenges is to improve the efficacy of nanoparticle therapeutic and to reduce the toxicity level. New treatment strategies are being explored with the rapid development in nanomaterials that has the potential to overcome existing problems using noble metallic nanoparticles. Prior to wide spread use, the impact on human health needs to be fully understood. Nanotechnology can play an excellent role in individualized medicine. Although more research is necessary for that. Noble metal nanoparticles can be effective in therapeutic and diagnostic agents as they show new properties at the atomic and supramolecular scales $(1-100 \mathrm{~nm})$.

It is true that there is increasing demand and use of nanomaterials in healthcare, industry, cosmetics. So, safety measures need to be taken to protect human health and surrounding environment. This clearly illustrates the need for more in depth studies on the safety profiles of metallic nanoparticles prior to healthcare use. Future research will need to investigate the use of metal nanoparticles for each potential human application. Overall gold, silver, platinum, selenium nanoparticles are ideally placed to make the transition from the laboratory bench top to the clinical field in the very near future. Metallic nanoparticles development is multidirectional and now it is widely used in cancer treatment. By these nanoparticles, a recent advance has been made the way in site-specific targeting. Metallic nanoparticle emphasizes their potency as new agent for future cancer therapeutic modalities. Are noble metal nanoparticles biocompatible or cytotoxic? So, it is important to test nanoparticle interactions experimentally and modify the nanoparticles for best biocompatibility with the cell in order to eliminate damage to healthy tissue. Against cancer, metallic nanomaterials have shown powerful tools. Though it need optimization and characterization for understanding their full potential. It is now high time to start transforming these platforms into clinical field to fight against cancer.

\section{References}

1. Daniel MC, Astruc D (2004) Gold nanoparticles: assembly, supramolecular chemistry, quantum-size-related properties, and applications toward biology, catalysis, and nanotechnology. Chemical reviews 104(1): 293346.

2. Sekar RP (2013) Formulation and evaluation of azathioprine loaded silver nanopartilces for the treatment of rheumatoid arthritis. Asian Journal of Biomedical and Pharmaceutical Sciences 3: 28.

3. Li X, Lan TH, Tien CH, Gu M (2012) Three-dimensional orientationunlimited polarization encryption by a single optically configured vectorial beam. Nature communications 3: 998.

4. The Lycurgus cup: transformation in glass, Belinda Crerar, curator. British Museum.

5. Weindling P (2000) Epidemics and genocide in Eastern Europe, 18901945. Oxford University Press, USA.

6. Apostolou P, Toloudi M, Chatziioannou M, Ioannou E, Knocke DR, et al. (2013) Anvirzel ${ }^{T M}$ in combination with cisplatin in breast, colon, lung, prostate, melanoma and pancreatic cancer cell lines. BMC Pharmacology and Toxicology 14: 18.

7. Akbar Vaseghi, Babak Bakhshinejd, Majide Sadeghizade, Reza Ashrafi Parchin (2014) Gold nanoparticles for biomedical applications. Young Researcher Club, Science and Researcher Branch, Islamic Azad University, Ardabil, Iran.

8. Li C, Shuford KL, Park Q, Cai W, Li Y, et al. (2007) High-Yield Synthesis of Single-Crystalline Gold Nano-octahedra. Angewandte Chemie 46(18): 3264-3268.

9. Granqvist CG, Buhrman RA (1976) Ultrafine metal particles. Journal of applied Physics 47: 2200-2219.

10. Metallic nanoparticles Dragomir Mirela (pdf), university of Nova Gorica, doctoral study, programme physics.

11. Rampino LD, Nord FF (1941) Preparation of palladium and platinum synthetic high polymer catalysts and the relationship between particle size and rate of hydrogenation. Journal of the American Chemical Society 63: 2745-2749.

12. Sinha A, Manjhi J (2015) Silver nanoparticles: green route of synthesis and antimicrobial profile. International Journal of Nanoparticles 8: 3050.

13. Hayat MA (2012) Colloidal gold: principles, methods, and applications. Elsevier.

14. Khan JA, Kudgus RA, Szabolcs A, Dutta S, Wang E, et al. (2011) Designing nanoconjugates to effectively target pancreatic cancer cells in vitro and in vivo. PLoS One 6(6): e20347.

15. Simard JM (2017) Synthesis of gold nanoparticles for biomacromolecular recognition. University of Massachusetts Amherst.

16. Turkevich J, Stevenson PC, Hillier J (1951) A study of the nucleation and growth processes in the synthesis of colloidal gold. Discussions of the Faraday Society 11: 55-75.

17. Brust M, Walker M, Bethell D, Schiffrin DJ, Whyman R (1994) Synthesis of thiol-derivatised gold nanoparticles in a two-phase liquid-liquid system. Journal of the Chemical Society, Chemical Communications 1994: 801-802.

18. Daniel MC, Astruc D (2004) Gold nanoparticles: assembly, supramolecular chemistry, quantum-size-related properties, and applications toward biology, catalysis, and nanotechnology. Chemical reviews 2004 104(1): 293-346. 
19. Brown KR, Walter DG, Natan MJ (2000) Seeding of colloidal Au nanoparticle solutions. 2. Improved control of particle size and shape. Chemistry of Materials 12: 306-313.

20. Bastús NG, Comenge J, Puntes V (2011) Kinetically controlled seeded growth synthesis of citrate-stabilized gold nanoparticles of up to 200 nm: size focusing versus Ostwald ripening. Langmuir 27: 11098-110105.

21. John De Britto A, Steena Roshan Sebastian (2013) Biosynthesis of silver nanoparticles and its antibacterial activity against human pathogens. International journal of Pharmacy and Pharmaceutical sciences 5(1): 257-259.

22. Sun T, Seff K (2011) Silver clusters and chemistry in zeolites. Chemical reviews 94: 857-870.

23. Hussain JI, Kumar S, Hashmi AA, Khan Z (2001) Silver nanoparticles: preparation, characterization, and kinetics. Adv Mat Lett 2(3): 188-194.

24. Soares JM, Cabral FA, de Araújo JH, Machado FL (2002) Exchange-spring behavior in nanopowders of CoFe 20 4-CoFe 2. Applied Physics Letters 98: 072502.

25. Patil S, Rajeshwari S, Rajiv P, Rajendran V, Seenivasan R (2015) Green synthesis of silver nanoparticle from leaf extract of Aegle marmelos and evaluation of its antibacterial activity. Int J Pharm Sci 7: 169-173.

26. Hussain JI, Kumar S, Hashmi AA, Khan Z (2011) Silver nanoparticles: preparation, characterization, and kinetics. Adv Mat Lett 2: 188-194.

27. Eustis S, Hsu HY, El-Sayed MA (2005) Gold nanoparticle formation from photochemical reduction of Au3+ by continuous excitation in colloidal solutions. A proposed molecular mechanism. The Journal of Physical Chemistry B 109(11): 4811-4815.

28. Iravani S (2011) Green synthesis of metal nanoparticles using plants. Green Chemistry 13: 2638-2650.

29. De Gusseme B, Sintubin L, Baert L, Thibo E, Hennebel T, et al. (2010) Biogenic silver for disinfection of water contaminated with viruses. Applied and Environmental Microbiology 76: 1082-1087.

30. Bowman MC, Ballard TE, Ackerson CJ, Feldheim DL, Margolis D M, et al. (2008) Inhibition of HIV fusion with multivalent gold nanoparticles. Journal of the American Chemical Society 130: 6896-7689.

31. Mallipeddi R, Rohan LC (2010) Nanoparticle-based vaginal drug delivery systems for HIV prevention. Expert opinion on drug delivery 7: 37-48.

32. Taylor U, Klein S, Petersen S, Kues W, Barcikowski S, et al. (2010) Nonendosomal cellular uptake of ligand-free, positively charged gold nanoparticles. Cytometry Part A 77(5): 439-446.

33. Lara HH, Ayala-Nuñez NV, Ixtepan-Turrent L, Rodriguez-Padilla C (2010) Mode of antiviral action of silver nanoparticles against HIV-1. Journal of nanobiotechnology 8: 1 .

34. Baram-Pinto D, Shukla S, Perkas N, Gedanken A, Sarid R (2009) Inhibition of herpes simplex virus type 1 infection by silver nanoparticles capped with mercaptoethane sulfonate. Bioconjugate chemistry 20: 1497-1502.

35. Papp I, Sieben C, Ludwig K, Roskamp M, Böttcher C, et al. (2010) Inhibition of influenza virus infection by multivalent sialic-acid-functionalized gold nanoparticles. Small 6(24): 2900-2906.

36. Sun L, Singh AK, Vig K, Pillai SR, Singh SR (2008) Silver nanoparticles inhibit replication of respiratory syncytial virus. Journal of Biomedical Nanotechnology 4: 149-158.

37. Tsai CY, Shiau AL, Chen SY, Chen YH, Cheng PC, et al. (2007) Amelioration of collagen-induced arthritis in rats by nanogold. Arthritis \& Rheumatology 56(2): 544-554.

38. Bhattacharya R, Mukherjee P, Xiong Z, Atala A, Soker S, et al. (2004) Gold nanoparticles inhibit VEGF165-induced proliferation of HUVEC cells. Nano Letters 4: 2479-2481.
39. Arvizo RR, Rana S, Miranda OR, Bhattacharya R, Rotello VM, et al. (2011) Mechanism of anti-angiogenic property of gold nanoparticles: role of nanoparticle size and surface charge. Nanomedicine: nanotechnology, biology and medicine 7(5): 580-587.

40. Ramezani N, Ehsanfar Z, Shamsa F, Amin G, Shahverdi HR, et al. (2008) Screening of medicinal plant methanol extracts for the synthesis of gold nanoparticles by their reducing potential. Zeitschrift für Natur for schung B 63: 903-908.

41. Chang AL, Khosravi V, Egbert B (2006) A case of argyria after colloidal silver ingestion. Journal of cutaneous pathology 33: 809-811.

42. Rahman MF, Wang J, Patterson TA, Saini UT, Robinson BL, et al. (2009) Expression of genes related to oxidative stress in the mouse brain after exposure to silver-25 nanoparticles. Toxicology letters 187: 15-21.

43. Moss AP, Sugar MD, Hargett MD (1979) The ocular manifestations and functional effects of occupational argyrosis. Arch Ophthalmol 97: 906908.

44. Chen W, Zhang Q, Kaplan BL, Baker GL, Kaminski NE (2014) Induced $\mathrm{T}$ cell cytokine production is enhanced by engineered nanoparticles. Nanotoxicology 8: 11-23.

45. Gottlieb NL, Smith PM, Penneys NS, Smith EM (1974) Gold concentrations in hair, nail, and skin during chrysotherapy. Arthritis \& Rheumatology 17: $56-62$.

46. Gottlieb NL (1983) Comparison of the kinetics of parenteral and oral gold. Scandinavian Journal of Rheumatology 12: 10-14.

47. Holister P, Weener JW, Vas CR, Harper T, Nanoparticles C (2003) Technology White Papers nr. 3. Cientifica Nanoporous Materials.

48. Feng QL, Wa J, Chen GQ, Cui KZ, Kim TM, et al. (2003) Antimicrobial activity of silver nanoparticles against bacterial species. Journal of Biomedical Materials Research B: 662-668.

49. Frens G (1973) Controlled nucleation for the regulation of the particle size in monodisperse gold suspensions. Nature physical science 241: 20-22.

50. Díaz MR, Vivas Mejia PE (2013) Nanoparticles as drug delivery systems in cancer medicine: emphasis on RNAi-containing nanoliposomes. Pharmaceuticals 6(11): 1361-1380.

51. Doria G, Conde J, Veigas B, Giestas L, Almeida C, et al. (2012) Noble metal nanoparticles for biosensing applications. Sensors 12(2): 1657-1687.

52. Qian X, Peng XH, Ansari DO, Yin Goen Q, Chen GZ, et al. (2008) In vivo tumor targeting and spectroscopic detection with surface-enhanced Raman nanoparticle tags. Nature biotechnology 26: 83-90.

53. Sperling RA, Parak WJ (2010) Surface modification, functionalization and bioconjugation of colloidal inorganic nanoparticles. Philosophical Transactions of the Royal Society of London A: Mathematical, Physical and Engineering Sciences 368: 1333-1383.

54. Ghosh P, Han G, De M, Kim CK, Rotello VM (2008) Gold nanoparticles in delivery applications. Advanced drug delivery reviews 60(11): 13071315.

55. Nishiyama N (2007) Nanomedicine: nanocarriers shape up for long life. Nature Nanotechnology 2(4): 203-204.

56. Chen H, Shao L, Ming T, Sun Z, Zhao C, et al. (2010) Understanding the photothermal conversion efficiency of gold nanocrystals. Small 6(20): 2272-2280.

57. Day ES, Morton JG, West JL (2009) Nanoparticles for thermal cancer therapy. Journal of biomechanical engineering 131(7): 074001.

58. Gibson JD, Khanal BP, Zubarev ER (2007) Paclitaxel-functionalized gold nanoparticles. Journal of the American Chemical Society 129(37): 11653-11661. 


\section{(c) (i) This work is licensed under Creative}

Submission Link: https://biomedres.us/submit-manuscript.php

$\begin{array}{ll}\text { BIOMEDICAL } & \text { Assets of Publishing with us } \\ \text { RESEARCHES } & \text { Global archiving of articles } \\ \text { - Immediate, unrestricted online access } & \text { Rigorous Peer Review Process } \\ & \text { - Authors Retain Copyrights } \\ & \end{array}$

